\title{
A GIANT PARTIALLY THROMBOSED AICA ANEURYSM
}

\author{
Eberval Gadelha Figueiredo', Marcos Q. T. Gomes², Rubens V. Brito-Neto', \\ Wellingson S. Paiva ${ }^{4}$, Manoel Jacobsen Teixeira ${ }^{5}$
}

Aneurysms of the anteroinferior cerebellar artery (AICA) are rare, comprising far less than $1 \%$ of all intracranial aneurysms. By 1989, only 33 cases had been reported in the literature ${ }^{1-4}$. Subsequently, numerous case reports and small series of AICA aneurysms have been reported $^{1,2,5-15}$. Giant aneurysms of proximal portion of AICA are even more uncommon, being difficult to estimate their actual incidence. These aneurysms constitute formidable surgical challenge and a judicious preoperative planning is mandatory since surgical nuances often determined the final outcome.

We present a case of giant partially thrombosed AICA aneurysm and discuss operative nuances required to minimize the morbidity associated with such surgically challenging lesions.

\section{CASE}

A 62 years old female presented sudden headache. At emergency room, neurological examination revealed meningismus. TC scan disclosed subarachnoid hemorrhage. Angiographic evaluation diagnosed three aneurysms at left middle cerebral artery, posterior communicating artery and a small left AICA aneurysm (Fig 1). Anterior circulation aneurysms were treated trough a left pterional approach. Postoperative course was uneventful. After two years, she returned complaining of trigeminal disestesias. Neurological examination displayed mild left facial paresis and right hemiparesis. TC scan revealed image suggestive of a thrombosed aneurysm compressing brainstem. This finding was confirmed by angiotomography and digital angiography that demonstrated giant thrombosed AICA aneurysm (Fig 2). Occlusion balloon test was carried out and patient stood asymptomatic after thirty minutes of occlusion.

A right transcocclear approach was performed and the facial nerve was skeletomized and mobilized. Vertebral arteries, basilar trunk and AICA were identified and dissected (Fig 3). Temporary clips were applied proximal and distally, aneurysm sac was incised. Thrombus was removed in order to deflate the aneurysm. Two fenestrated clips were applied to the neck to preserve AICA (Fig 4). Two temporary clipping (thirty minutes each) were necessary to performing these maneuvers. Abdominal fat pad was harvested and used to pack the petrosal space and a catheter for lumbar drainage was inserted.

Definitive deficits were deafness in the right side and right facial paresis. Postoperative angiogram disclosed adequate surgical result (Fig 5). The patient has agreed with this case publication.

\section{DISCUSSION}

The surgical exposure of AICA aneurysms is difficult due to distance of the lesions from the cranial base and their close relationship to the brainstem and lower cranial

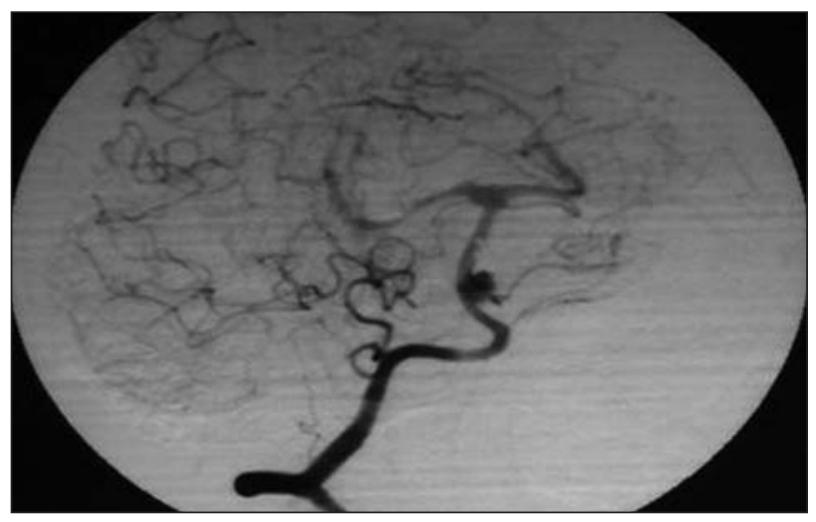

Fig 1. Vertebral angiogram depicting a small left AICA aneurysm.

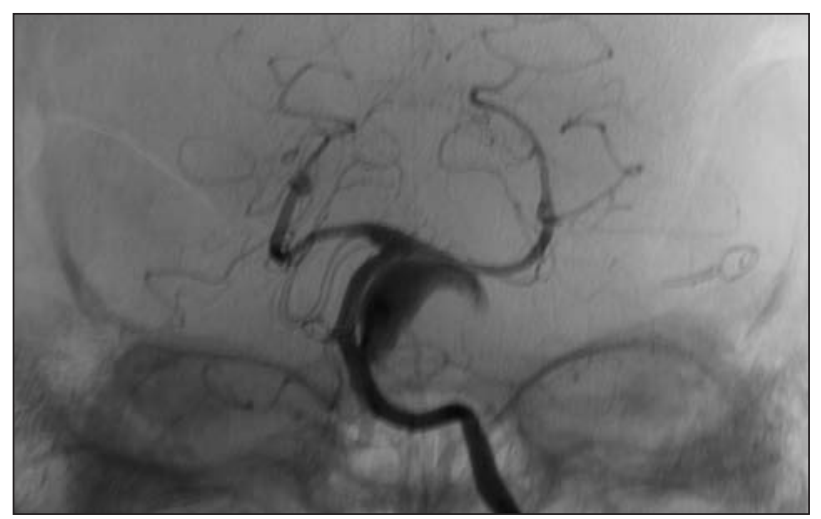

Fig 2. Vertebral angiogram confirms angiotomography findings. A giant partially thrombosed AICA aneurysm is evidenced.

\section{ANEURISMA GIGANTE TROMBOSADO DA AICA}

Division of Neurological Surgery, University of São Paulo School of Medicine, São Paulo SP, Brazil: 'Supervisor and Cerebrovascular Surgery Coordinator; ${ }^{2}$ Skull Base Surgery Coordinator; ${ }^{3}$ Assistant Professor, Division of Otorhinolaringology; ${ }^{4}$ Resident; ${ }^{5}$ Director and Chairman.

Received 31 January 2008, received in final form 11 April 2008. Accepted 16 May 2008.

Dr. Eberval G. Figueiredo - Rua Oscar Freire 1456/34 - 05409-010 São Paulo SP - Brasil. E-mail: ebgadelha@yahoo.com 


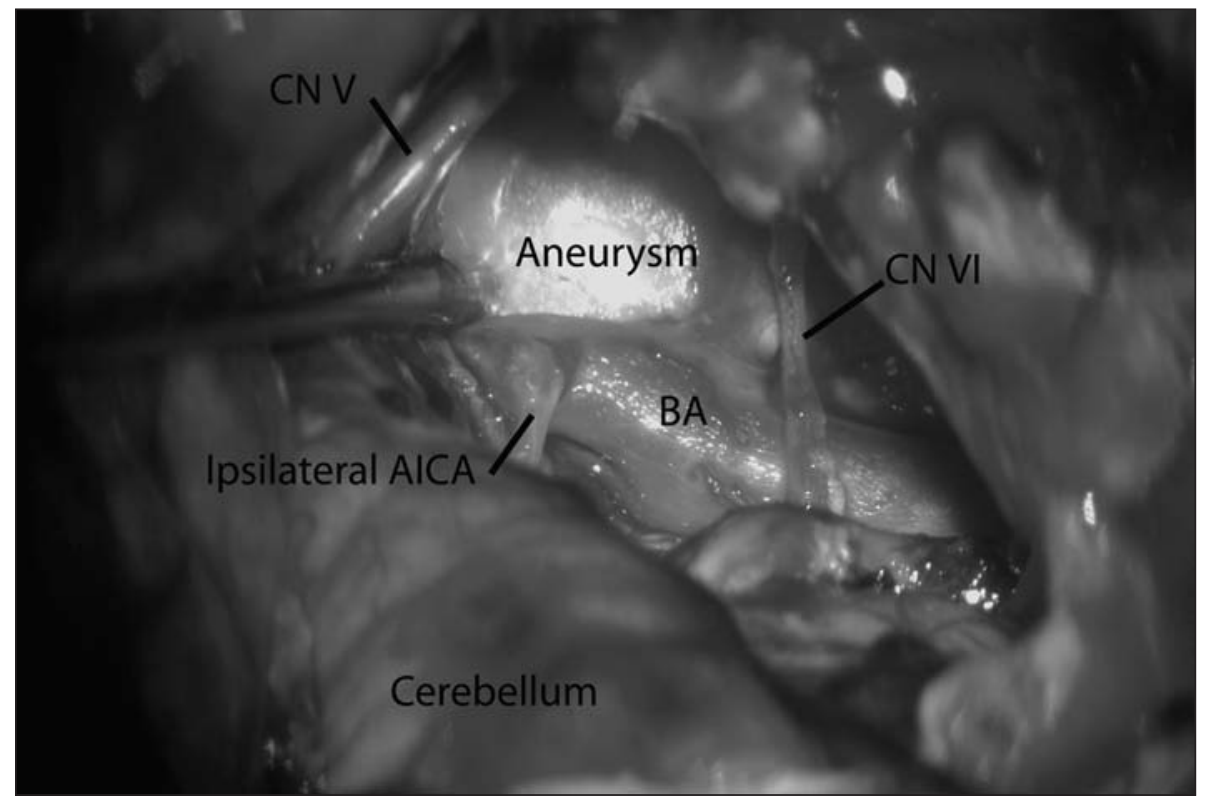

Fig 3. A transcoclear approach has been performed. Dura mater has been opened and facial nerve has been mobilized. Tentorium has been cut and basilar artery trunk (BA) has been dissected and exposed. Giant aneurysm is visualized with its neck originating from AICA, between cranial nerves $V$ and $\mathrm{VI}(\mathrm{CN} V ; \mathrm{CN} V \mathrm{VI}$.

Fig 4. After temporary clipping, aneurysm has been deflated and two definitive fenestrated clips have been applied to exclude aneurysm from circulation.

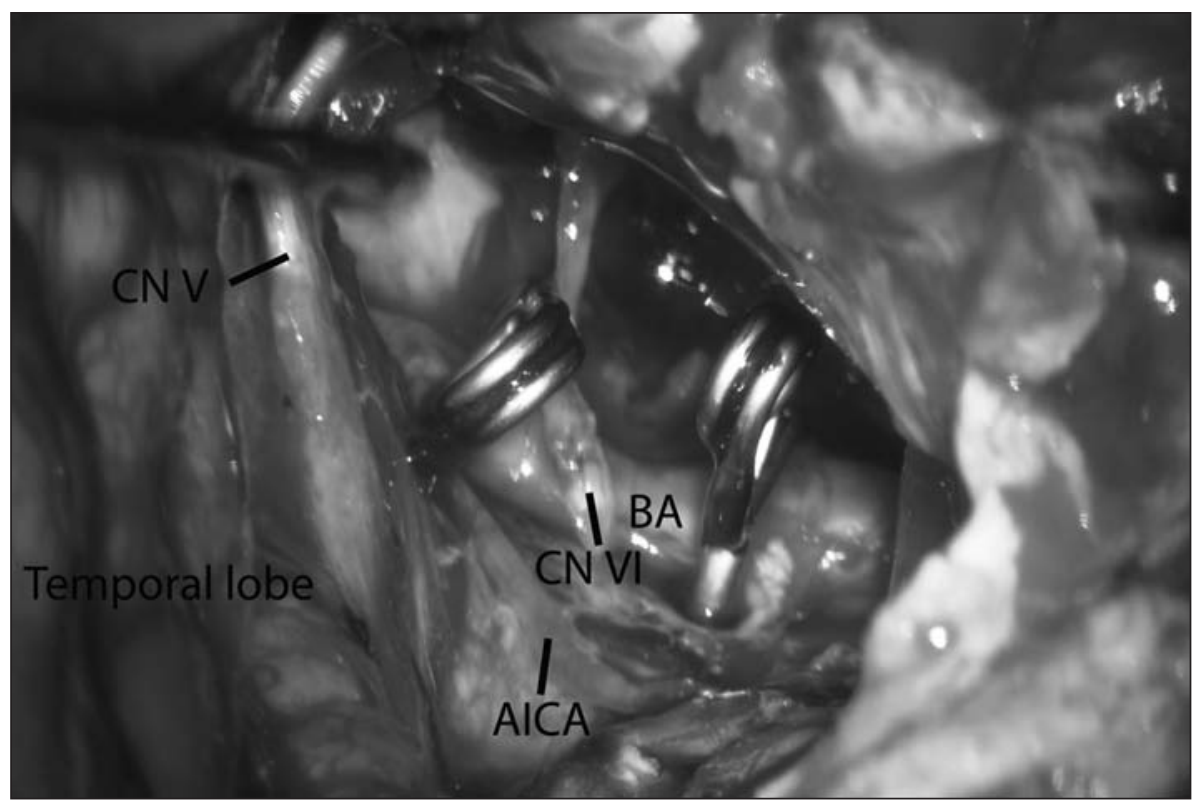

nerves ${ }^{3}$. Giant aneurysms displace the pons, making surgical access difficult. AICA aneurysms are located ventral to the brainstem, where the artery emerges from the basilar artery, or on the lateral surface of the cerebellopontine fissure. When selecting a technique, two important factors must be considered: the location of the aneurysm with respect to the clivus and along the course of the parent artery ${ }^{3}$.

The retrosigmoid approach is associated with minimal intrinsic complications compared with transpetrosal techniques ${ }^{3}$. Skeletonizing the transverse sigmoid junction, including the anterior aspect of the sigmoid sinus, allows the sinus to be retracted laterally to obtain extra space, minimizing retraction over the cerebellum. It of- fers a straight corridor between the cranial nerves (Vth and VIIth) to access the origin of the AICA from the basilar artery ${ }^{3}$.The retrosigmoid approach offers an excellent route to distal aneurysms on the postmeatal or meatal segments of the artery ${ }^{3,9}$. It is not suitable to approach giant proximal AICA aneurysms. In such cases, more "dramatic" approaches are needed ${ }^{13-15}$.

The drawbacks of transpetrosal techniques arise from the intrinsic side effects associated with these techniques (e.g., facial nerve palsy, deafness, CSF leakage) ${ }^{3}$. We opt for transpetrosal approaches in selected cases with giant aneurysms. Deflating an aneurysm and rendering it pulseless facilitates dissection from the ventral surface of the 


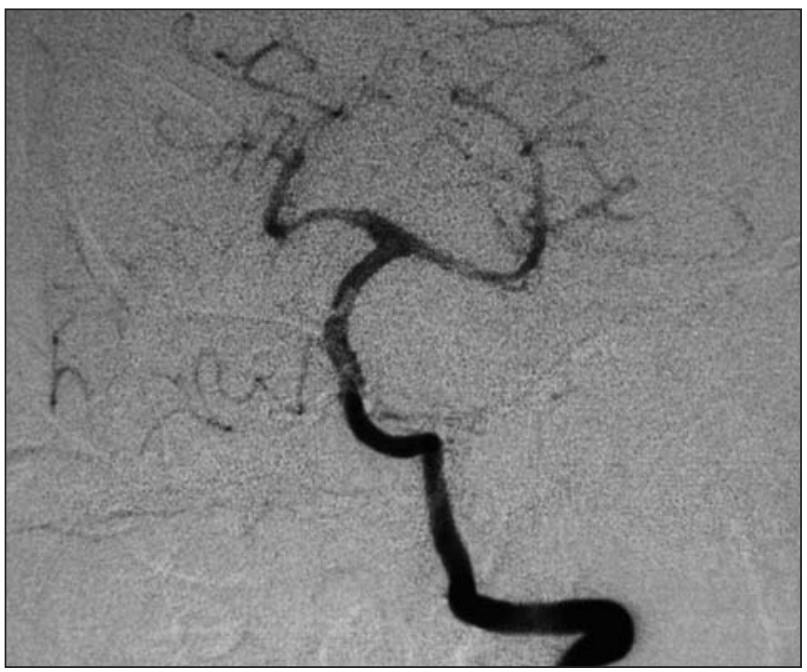

Fig 5. Postoperative angiogram depicts complete clipping of the aneurysm without stenosis of the basilar artery.

pons and isolates the aneurysm from the perforators to ensure adequate clipping ${ }^{3}$.

The complication rate of almost $60 \%$ indicates the complexity of these lesions ${ }^{3}$. Given that the AICA originates ventral to the brainstem and cranial nerves, the most common complication is related to injury of the cranial nerves, especially the VIth cranial nerve. Compromise of the long tracts possibly indicates brainstem ischemia during dissection of the perforators ${ }^{3}$. After posterior fossa surgery and transpetrosal approaches, CSF leakage is common ${ }^{3}$. The incidence of CSF leaks can be decreased by optimizing the dural closure. The closure can be covered with strips of fat harvested from the abdomen, and fibrin glue can be applied to seal dural defects ${ }^{3}$. Tight soft tissue approximation is paramount for preventing CSF leaks. The use of lumbar drains after surgery also helps avoid a CSF leak ${ }^{3}$.

Definitive morbidity is associated with the surgical route. Hearing loss is universal finding following transcocclear approach, as well as paresis of facial nerve. We consider the occlusion test important for the good outcome of this patient. It ensured that patient would tolerate at least thirty minutes of temporary clipping during surgery. This information provides us confidence while we were working to secure the aneurysm and save parent and efferent vessels. Furthermore, if the aneurysm proved to be unclippable, a reverse flow technique, with occlusion of proximal basilar artery, might be tempted.

Most authors do not recommend coil embolization for large or giant aneurysms that constrict the brainstem ${ }^{3}$. Aggressively packing an aneurysm with coils may exert additional mass effect ${ }^{3}$. Provided that the high rates of recurrence associated with coiling large and giant aneu- rysms, endovascular treatment ${ }^{8}$ may be reckoned as an alternative treatment only for selected cases $^{3}$.

Deliberate basilar or vertebral artery occlusion is a strategy used to reduce proximal blood flow to treat aneurysms when clipping is not possible ${ }^{3}$. The ultimate goal is to achieve thrombosis. The most feared complication is progressive uncontrolled thrombosis of the parent vessel. Acute or delayed vascular insufficiency distal to the occlusion is also a concern ${ }^{3}$. Extracranial-intracranial bypass is indicated only for giant aneurysms that require a reduction in blood flow through the source vessel (reverse-flow technique). In such cases, the aneurysm exerts mass effect against the brainstem, which must be decompressed, and the circulation distal to the aneurysm is compromised. Before a parent vessel is occluded, blood flow to the territory distal to the occlusion must be demonstrated ${ }^{3}$, as was indicated by the occlusion balloon test in the present case.

\section{REFERENCES}

1. Locksley HB, Sahs AL, Knowler L. Report on the Cooperative Study of Intracranial Aneurysms and Subarachnoid Hemorrhage: Section II. General survey of cases in the central registry and characteristics of the sample population. J Neurosurg 1966;24:922-932.

2. Locksley HB. Natural history of subarachnoid hemorrhage, intracranial aneurysms and arteriovenous malformations: based on 6368 cases in the cooperative study. J Neurosurg 1966;25:219-239.

3. Gonzalez LF, Alexander MJ, McDougall CG, Spetzler RF. Anteroinferior cerebellar artery aneurysms: surgical approaches and outcomes--a review of 34 cases. Neurosurgery 2004;55:1025-1035.

4. Kamii H, Ogawa A, Sakurai Y, Kayama T. Anterior inferior cerebellar artery aneurysm with a sudden onset of caudal cranial nerve symptoms. No Shinkei Geka 1989;17:387-391.

5. Banczerowski P, Sipos L, Vajda J. Aneurysm of the internal auditory artery: our experience and review of the literature. Acta Neurochir (Wien) 1996;138:1157-1162.

6. Chen ZP. Anterior inferior cerebellar artery aneurysm: report of two cases . Zhonghua Wai Ke Za Zhi 1990;28:490-511.

7. Crockard HA, Koksel T, Watkin N. Transoral transclival clipping of anterior inferior cerebellar artery aneurysm using new rotating applier: technical note. J Neurosurg 1991;75:483-485.

8. Gruber A, Killer M, Bavinzski G, Richling B. Clinical and angiographic results of endosaccular coiling treatment of giant and very large intracranial aneurysms: a 7-year, single-center experience. Neurosurgery 1999;45:793-803.

9. Guzman R, Grady MS. An intracranial aneurysm on the feeding artery of a cerebellar hemangioblastoma: case report. J Neurosurg 1999;91:136-138.

10. Ildan F, Gocer AI, Bagdatoglu H, Uzuneyupoglu Z, Tuna M, Cetinalp E. Isolated trigeminal neuralgia secondary to distal anterior inferior cerebellar artery aneurysm. Neurosurg Rev 1996;19:43-46.

11. Kyoshima K, Matsuda M, Handa J. Cerebral aneurysm of the distal anterior inferior cerebellar artery: case report. Nippon Geka Hokan 1995;64:139-145.

12. Lawton MT, Daspit CP, Spetzler RF. Technical aspects and recent trends in the management of large and giant midbasilar artery aneurysms. Neurosurgery 1997; 41:513-521.

13. Figueiredo EG, Zabramski JM, Deshmukh P, Crawford NR, Preul MC, Spetzler RF. Anatomical and quantitative description of the transcavernous approach to interpeduncular and prepontine cisterns: technical note. J Neurosurg 2006;104:957-964.

14. Siwanuwatn R, Deshmukh P, Figueiredo EG, Crawford NR, Spetzler RF, Preul MC. Quantitative analysis of the working area and angle of attack for the retrosigmoid, combined petrosal, and transcochlear approaches to the petroclival region. J Neurosurg 2006;104:137-142.

15. Figueiredo EG, Zabramski JM, Deshmukh P, Crawford NR, Spetzler RF, Preul MC. Comparative analysis of anterior petrosectomy and transcavernous approaches to retrosellar and upper clival basilar artery aneurysms. Neurosurgery 2006;58(Suppl 1):ONS13-21. 